\title{
DISCO: Interference-Aware Distributed Cooperation with Incentive Mechanism for 5G Heterogeneous Ultra-Dense Networks
}

\author{
Chungang Yang*, Jia Xiao*, Jiandong $\mathrm{Li}^{*}$, Xiaoqiang Shao*, Alagan Anpalagan ${ }^{\dagger}$, Qiang $\mathrm{Ni}^{\ddagger}$, Mohsen Guizani $^{\S}$
}

\begin{abstract}
Interference and traffic imbalance hinder the improved system performance in heterogeneous ultra-dense networks. Network cooperation becomes a promising paradigm with sophisticated techniques which can significantly enhance the performance. In this article, a coalition game-theoretic framework is introduced to characterize the cooperative behaviors, and thus exploring these cooperative benefits and diversity gains. First, we introduce the basis of the coalition games, and then we survey its latest applications, in particular, the interference mitigation and traffic offloading. Different from most of the current applications, we concentrate on the cooperative incentive mechanism design since the node cooperation always means resource consumption and other costs. Moreover, for the incentive mechanism, a cooperative spectrum leasing is introduced. To mitigate the interference and balance traffic, we propose two schemes under the presented framework: interference alignment with spectrum leasing (IASL) and traffic offloading with spectrum leasing (TOSL). Simulation results show the improved performance of the cooperative gains using the proposed IASL and TOSL schemes.
\end{abstract}

Index Terms-Coalition game; 5G; Interference alignment; Incentive mechanism; Traffic offloading; Ultra-dense networks.

\section{INTRODUCTION}

The 5G mobile communication systems should be spectrum and energy efficient to realize the 1000-fold capacity improvement in a cost-effective way. Internet of things (IoT) and mobile Internet are becoming the main driving forces. Exponential growth in data traffic, billions of connections, and densification of small cells are the main characteristics that a fully mobile and connected community demands. Ultra-dense networks (UDNs) underlaying macrocells are identified as one of the best means to improve the system capacity, extend

C. Yang, J. Xiao, J. Li, and X. Shao are with the State Key Laboratory on Integrated Services Networks, Xidian University, Xi’an, 710071 China (cgyang@mail.xidian.edu.cn; jiaxiao@stu.xidian.edu.cn; jdli@mail.xidian.edu.cn; 2990839145@qq.com).

$\dagger$ A. Anpalagan is with the Department of Electrical and Computer Engineering, Ryerson University, Toronto, ON, Canada (email: alagan@ee.ryerson.ca).

$\mp$ Q. Ni is with School of Computing and Communications, Lancaster University, Lancaster, UK. (q.ni@lancaster.ac.uk)

$\S$ M. Guizani is with Electrical and Computer Engineering Department University of Idaho, Idaho, USA. (email: mguizani@ieee.org).

This work was supported in part by the National Science Foundation of China under Grant 61231008; by the Special Financial Grant from the China Postdoctoral Science Foundation (2016T90894); by the Special Financial Grant from the Shaaxi Postdoctoral Science Foundation (154066); by the CETC Key Laboratory of Data Link Technology(CLDL-20162309); by the Natural Science Basic Research Plan in Shaanxi Province of China (2017JZ021); by the ISN02080001; by the 111 Project under Grant B08038; and by the Shaanxi Province Science and Technology Research and Development Program (2011KJXX-40). the coverage, and enhance the quality of experience (QoE) of subscribers.

However, the problems of interference and traffic imbalance limit the achievable performance and the capabilities of the heterogeneous small cells. For instance, most subscribers will associate to the macrocell due to the larger downlink power, even if the subscribers are close to a specific small cell, thus fewer users associate to small cells. Therefore, the potential capabilities of small cells cannot be fully exploited due to their intrinsic characteristics, e.g., the small cells' lower downlink power and less antenna gains [1], [2]. This leads to a serious traffic imbalance problem. Meanwhile, the intensive deployment of small cells produces a severe interference problem with the limited spectrum resources in the hotspots.

Although interference and traffic imbalance problems will be more serious with a huge number of small cells, there exist more cooperation opportunities among them. Cooperative diversity gains can be exploited by forming potential cooperative coalitions to well deal with these technical problems. Extensive cooperative framework and technology have been studied to improve the cooperation gains, e.g., overlapping coalition framework, coordinated multiple points transmission/reception (CoMP), and cell range expansion (CRE). However, cooperation and coordination always mean additional resource consumption and other related cost, such as power consumption, necessary information interaction, and signaling overhead. Therefore, it is important to design a suitable incentive mechanism to motivate such cooperative coalition formation.

In this article, the cooperative behaviors of the small cells are characterized by introducing a coalition game-theoretic framework, and thus exploring these cooperative benefits and diversity gains. Although coalition games have found applications in wireless communication networks, an incentive mechanism is important to form the cooperative coalitions.

The remainder of this paper is organized as follows. A summary of the technical challenges and opportunities is given in section II. Section III presents the basics of a coalition game. In section IV, we briefly introduce a cooperative framework with an incentive mechanism. In section V, two applications of the proposed coalition game framework with an incentive mechanism are presented with simulation results showing the performance gains. The article is concluded in section VI. 


\section{Technical Challenges and Opportunities: Node CoOperation And COOPERATIVE GAME}

Heterogeneous UDNs with node cooperation are recognized as the most powerful $5 \mathrm{G}$ framework trends for both the mobile Internet [3], [4] and IoT [5]. For instance, the c-radio access network, where c stands for centralized, collaborative and cloud, and CoMP technologies, has been applied to improve network performance and achieve load balance [1], [7]. To optimize the utilization of densely deployed nodes and smoothly advance towards 5G networks, an Internet-oriented architecture of mobile networks is proposed in [3]. Meanwhile, authors in [4] point out that UDNs are the new paradigm with network cooperation and availability of higher density of radio nodes. To reduce the cost, device-to-device communications can offload the network and ensure the ubiquity of high quality services. In addition, to meet the technical and economical requirements of exponentially growing machine-type communications (MTC), authors in [5] advocated the use of small cells to handle the massive and dense MTC rollout. These call for a cooperative framework for the $5 \mathrm{G}$ heterogeneous UDNs to meet the trends for both the mobile Internet [3], [4] and the IoT [5].

1) Technical Interference and Traffic Imbalance Challenges: We concentrate on traffic imbalance and mutual interference to enhance both energy and spectrum efficiency. The spectrum resources are scarce and valuable, and resource coordination has been well studied from academia, industry, and standardized bodies [1].

There are two kinds of interference management schemes including resource coordination-based and interferenceoriented ones. On one hand, the resource coordination-based scheme at the transmitter side [11], [12] will try to avoid interference by partially orthogonally allocating or fully reusing the spectrum resources, which can be implemented in frequency, time, space, and power domains, such as almost blank subframes, CoMP and enhanced inter-cell interference coordination (eICIC). On the other hand, there are interference cancellation techniques at the receiver side, for instance, successive interference cancellation [9] and IA [10]. These kinds of interference mitigation schemes will reconstruct the interference or exploit the interference characteristics from the spacial perspective like zero-forcing precoding, and then eliminate it [11].

The above inter- and intra-tier interference problems also result in traffic imbalance problems, because of the featured different downlink powers and antenna gains. That is, most of the small cells underlaying macrocells cannot be effectively associated due to the much larger downlink power and antenna gains of macrocells. During the past decades, various schemes for traffic offloading have been proposed or standardized, for instance, mobility load balancing (MLB) [2], CRE, capacity offloading [13], [14], and dynamic association.

2) Potential Node Cooperative Opportunities: Network cooperative methodologies have found extensive applications in both interference mitigating and energy saving. In fact, interference management schemes such as eICIC, CoMP and IA, and traffic offloading schemes such as MLB are implemented among different cells involving the cooperation among them. Cooperative diversity gains have great potentials for reducing energy consumption of mobile users [2]. However, different players are diverse with heterogeneous properties including the capabilities of QoE provisions, rationality towards preference, and dynamic association behaviors. That is why the cooperative game theory has been widely applied to model, analyze, and explore these cooperative diversity gains, characterize behaviors of rational players, and design distributed algorithms [8].

3) Cooperative Games: In general, bargaining games and coalition games are two types of cooperative games, which have extensive applications. Classical bargaining games describe the bargaining process to agree on a cooperation protocol [6], while coalition games describe the coalitions formed by cooperative groups of players [8]. However, motivating cooperation among different cells means additional resource consumption and other related performance cost. Therefore, it is important to design a suitable incentive mechanism to motivate such cooperative coalitions.

\section{COAlition Game TheOry}

The basics of a coalition game is introduced in this section, where we concentrate on the basic concepts, principles, characteristics, classifications, and solutions.

In principle, coalition games contain a set of players, and they aim to strengthen their positions by forming cooperative coalitions. The optimal strategic decision-making and the dynamic behaviors of each player in the game always interact with each other. Different from non-cooperative games in which players independently pursue their own payoff maximization or cost minimization in a selfish manner, players in coalitional games emphasize social optimality and rationality to optimize the payoff distributions among different players. Meanwhile, coalition games concentrate on system efficiency and individual fairness among different players. In addition to these interactive and social rational players, the coalition value is another key concept of a coalitional game, which quantifies the value of a coalition. If the coalition value can be represented by a scalar featured function, then the coalitional games are named as the coalitional games with transferable utilities. This kind of scalar featured function is also called the characteristic function or payoff function, which quantifies the value of each coalition.

The form and type of a game are determined by the definition of the coalition value. Nevertheless, a coalition game is determined uniquely, independent of the value definition. Along with the category of coalitional games with transferable utility, depending on whether the payoff function is impacted by the other members from other coalitions, thus resulting in the characteristic games and formation games. For the former, the coalition payoff of the characteristic games is solely determined by the members in the considered coalition, and it is not dependent on the other members from any other coalition. In practice, formation games are most popular due to the existing interactions among different players.

Coalitional graph games, coalition formation games and canonical coalitional games are introduced from their potential 
applications in wireless communication networks [8]. Different solutions including core, Shapley value, and nucleolus are presented assuming that the grand coalition is formed because of super-additive property. However, coalition formation games intend to provide solutions to what is the optimal size of coalition subjected to changes in the number of players in the coalition and physical constraints. In the following sections, we concentrate on the coalition formation games with characteristics of no super-additive property in the partition form, and we also concentrate on the cost effects on forming coalitions.

\section{COOPERATIVE FRAMEWORK WITH AN INCENTIVE MECHANISM}

In this section, we first survey the recent applications of coalition games. Then, we summarize the motivations of introducing incentive mechanism design, which includes the IA [10], inter-tier traffic offloading and spectrum leasing.

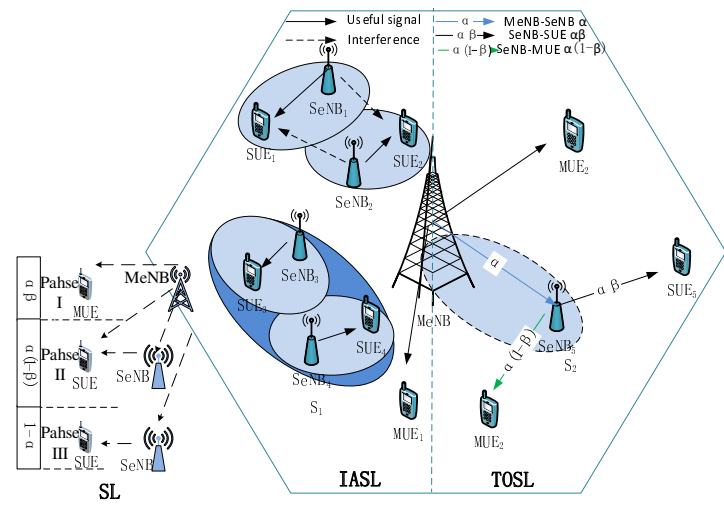

Fig. 1. Cooperative framework with an incentive mechanism with IA and traffic offload as two applications.

A heterogeneous UDN is illustrated in Fig. 1. For instance, in a crowded stadium or an open-air assembly, one can expect intensive deployment of multiple small cells evolved base stations (SeNBs), such as the femtocell base stations, microcell base stations, picocell base stations, and so on. SeNBs are assumed to fully share the frequency resources licensed to the macrocell evolved base station (MeNB). In addition, we assume that both MeNB, SeNBs, and their associated user equipment, for instance, small cell user equipment (SUEs), and macrocell user equipment (MUE) are with multiple antennas. There exist both inter-tier interference between SeNBs and $\mathrm{MeNB}$, and intra-tier interference among different SeNBs.

The UDN leads to a more complex intra- and inter-tier interference and traffic offloading, which enormously affects the network performance and user experience. Different SeNBs and MeNB can form cooperative coalitions, which include homogeneous and heterogeneous coalitions, to mitigate interference and offload traffic according to the revenue and the traffic situation of the adjacent interfering SeNBs, such as the $\left\{\mathrm{SeNB}_{3}, \mathrm{SeNB}_{4}\right\}$ and $\left\{\mathrm{MeNB}, \mathrm{SeNB}_{5}\right\}$ in Fig. 1. Moreover, the spectrum leasing is shown in Fig. 1. We normalize each time-slot to one unit in length and divide it into three phases. In phase I, only the MeNB transmits its packets to the MUE with the length $\alpha \beta$; In phase II, the MeNB and SeNBs simultaneously transmit with a coordinated transmission with the length $\alpha(1-\beta)$. In phase III, the MeNB stops its transmission and leases the spectrum to the related coalition of SeNBs with the length $(1-\alpha)$, where SeNBs transmit using the distributed IA. With these implementations, the cooperation of the coalition has brought much corresponding benefits, such as the enhancement of spectrum efficiency by heterogeneous coalitions and the interference avoidance by the homogeneous coalitions.

\section{A. Interference Alignment}

IA is a powerful interference mitigation technique. It involves the coordination among different antennas of one node, or different virtual multiple antennas of multiple nodes. Basics of IA can be summarized as follows: the transmitter pre-codes the message using the channel state information at the receiver side, and the beam forming is implemented to suppress the interference. The interference coming from a different transmitter is forced to be aligned in particular dimensions of a signal such that the remaining dimensions become free of interference, thus improving the system performance.

In Fig. 1, different non-cooperative SeNBs are experiencing strong intra-tier interference. If IA is implemented, then the intra-tier interference will be mitigated, thus cooperatively improving the spectrum efficiency. Most of the current literature is researched from the signal processing perspective, and they focus on how to design the effective pre-codes [9]. However, there lacks an efficient coordination between the transmitter and the interferer, e.g., the antennas or the virtually cooperative nodes. It is also necessary to answer the questions of who will participate in the interference alignment coalition and why? How about the performance metric involving coalition formation cost, the interference-aware, the received signal strength indication (RSSI) or the location-based?

Coalition formation games have found several applications to answer these questions. For instance, a coalition game was used to formulate the SeNBs' cooperative behaviors in [9], where the IA techniques are implemented by the cooperative SeNBs to enhance their transmission rate in each coalition. The payoff function encapsulates the profit from cooperation with regard to an increased data rate and the cost with regard to pilot signal transmission power during channel estimation.

In this article, we jointly concentrate on two kinds of interference management schemes including resource coordinationbased [11] and interference-oriented ones [9], [10], which have been separately studied. In this article, we will jointly design the interference management in the coalition games with an incentive mechanism.

\section{B. Inter-Tier Traffic Offloading}

Observed from the Fig. 1, if the $\mathrm{SeNB}_{5}$ is in the open access mode, then the MUE can be offloaded to the $\mathrm{SeNB}_{5}$ with a effectively received power and a weaken interference power. This will significantly improve the SINR performance of the MUE, and as payback, the MeNB can lease parts of its channels. Traffic offloading is one of the best ways to 
solve the traffic imbalance problem between the macrocell and small cells. Traffic offloading always involves the problems of what should be offloaded, where to offload, and so on. This process can be modeled as a game of different players, e.g., the macrocell and small cells.

The authors in [13] proposed an energy-aware traffic offloading method between multiple SeNBs and MeNB, which was modeled as a Nash bargaining game. To attain an optimal win-win for both SeNBs and MeNB, mutual interference compensation, interference-related right selected target-SeNB and an energy-aware trigger of source-MeNB were provided in [13], which helped to attain more dimensions of cooperation gains and diversity. In [14], to model data offloading, a queuing-theoretic model was formulated with the elastic data flows notion, or more explicitly, cell range expansion together with inter-cell interference coordination. However, as the delay increases, the users' satisfaction sharply decreases.

In summary, coalition formation games study and analyze the behaviors and interactions among nodes during various kinds of cooperation and coordination, which also facilitate the self-organizing, decentralized, and autonomic networks. However, motivating cooperation among different nodes means additional resource consumption and other related performance cost. Therefore, it is important to design a suitable incentive mechanism to motivate such cooperative coalitions. In this article, we present a coalition game-theoretic framework to characterize the cooperative behaviors with the spectrum leasing as the incentive mechanism.

\section{Spectrum Leasing as an Incentive Mechanism}

Spectrum leasing is used as advanced spectrum resource coordination to improve the spectrum efficiency; meanwhile, it can improve spectrum utilization flexibility. By now, three dimensions of spectrum leasing have been studied from frequency channel, time slot, and spatial perspectives [12]. For instance, the spectrum leasing was proposed as the incentive mechanism to motivate the SeNBs for open access to offload traffic of MeNB in [12]. In return, the MeNB would coordinate the downlink power to relieve the interference as the compensation to the SeNBs. A coalitional game framework was proposed between the MeNB and the SeNBs, where a small cell user may act as a relay for the macrocell users. In return, each cooperative macrocell user grants the small cell user a fraction of its superframe.

\section{Two Applications with InCEntive MeChanism}

\section{A. IA with Spectrum Leasing (IASL)}

Both inter- and intra-tier interference enormously reduce the performance and hinder the intensive deployment of the network. Promisingly, various kinds of technologies are studied to mitigate the interference at the transmitter or the receiver side. With the development of advanced cooperation networking paradigms, the nodes can form clusters by cooperating to coordinate their transmission with some sustainable constraints. Moreover, many studies lack the motivation to drive the cooperation among nodes. Thus, the spectrum leasing was combined as the incentive mechanism to boost the cooperation to perform IA.
1) Details of Proposed IASL Scheme: We present a joint resource management and IA scheme to optimize the revenue of each small cell, the pseudo-code can be found in algorithm 1. We consider the downlink transmission of an Orthogonal Frequency Division Multiple Access (OFDMA) macrocell network such as an LTE advanced macro-cell. Initially, the initial coalitional structure consists of non-cooperative SeNBs. All SeNBs fully share the spectrum of the MeNB and transmit with the maximum power. Firstly, all SeNBs collect the reference signal receiving powers (RSRPs) and the cell identifier (ID) from the neighboring SeNBs by their respective UEs. Based on the collected information, each SeNB constructs the interference list in a decreasing sequence. Then, each SeNB computes the current utility $u$, where the utility $u$ is a scalar function of the SeNB' total transmission rate.

From the top of the list, each SeNB computes the cooperation cost $c=\frac{v \sigma^{2}}{\eta}$, where $c$ is the cost of power consumption that SeNB transmit a pilot tone to the farthest SeNB's SUE of the coalition. The terms $v, \sigma^{2}$, and $\eta$ are the SINR threshold, the background noise power, and the channel gain between the SeNB with its farthest coalition SeNB, respectively. Each SeNB selects the potential cooperator from the top of the list to coordinate their transmissions by comparing the revenue and the cost. When the IA conditions are satisfied [9], the SeNB proposes cooperation with interfering SeNB. Next, MeNB determines to implement the spectrum leasing and power control. Here, the revenue of SeNB is defined as $U=w f_{p}\left(R_{S}\right)-C_{0} P$, where the revenue is defined as the data transmission benefits minus the leasing payment. The term $w$ is a weight factor to tradeoff the benefits and payment, and $f_{p}(x)$ is the function of SeNB user with respect to data transmissions. $R_{S}=\alpha(1-\beta) R^{I I}+(1-\alpha) R^{I I I}, R^{I I}$ and $R^{I I I}$ are the SeNB' transmission rate in the phase II and the phase III of the spectrum leasing process. The detail of spectrum leasing process refers to the description in Fig. 1. $C_{0}$ is the unit price for SeNB's power. This function can be approximated as a convex function by fixing other parameters, then the leasing coefficient $\alpha, \beta$ and transmitted power $P$ can be solved, respectively. Finally, each coalition implements the corresponding operation, including IA and spectrum leasing, to improve the performance.

2) Simulation Results: Simulation is conducted using Matlab 2014b to assess the performance of the proposed IASL algorithm. We consider a heterogeneous cellular network, where multiple small cells are randomly distributed within a single hexagonal macrocell with a radius of $1 \mathrm{Km}$. The number of small cells is 50-300. The maximum downlink transmission powers are $46 \mathrm{dBm}$ and $20 \mathrm{dBm}$ for the macrocell and small cells, respectively. Here we use the femtocell as one example. Certainly, the proposed scheme can be extended to other types of small cells, for instance, the picocells. We assumed both the nodes and user equipments are with 4 antennas, which can help to well apply powerful interference mitigation capabilities of IA. Meanwhile, each femtocell only serves one user in the closed access way. The distance dependent path loss shadowing affects the transmissions according to $3 \mathrm{GPP}$ specifications [15].

Fig. 2 illustrates the improved cooperative gains of the pre- 


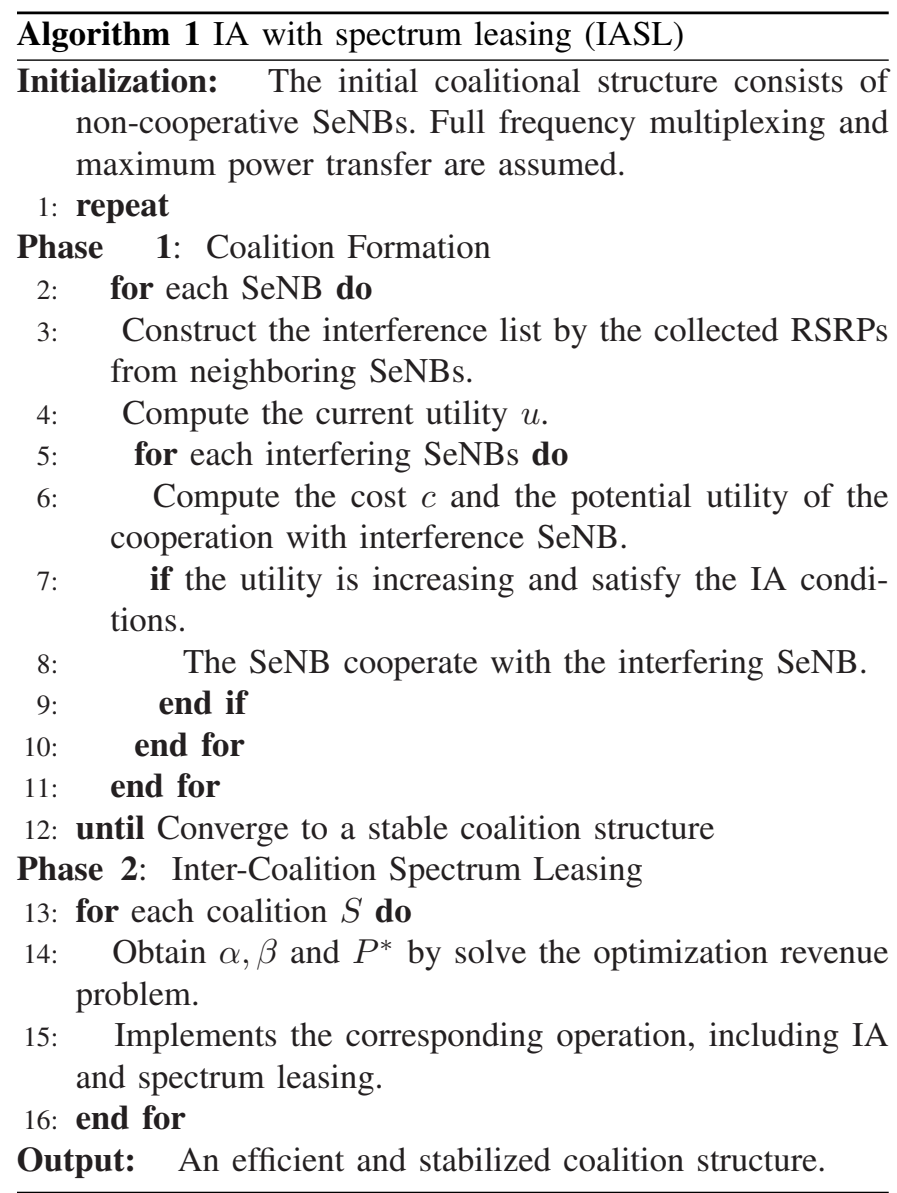

sented IASL scheme compared to that of the non-cooperation scheme. The non-cooperation scheme is implemented among the SeNBs without the resource coordination and spectrum leasing.

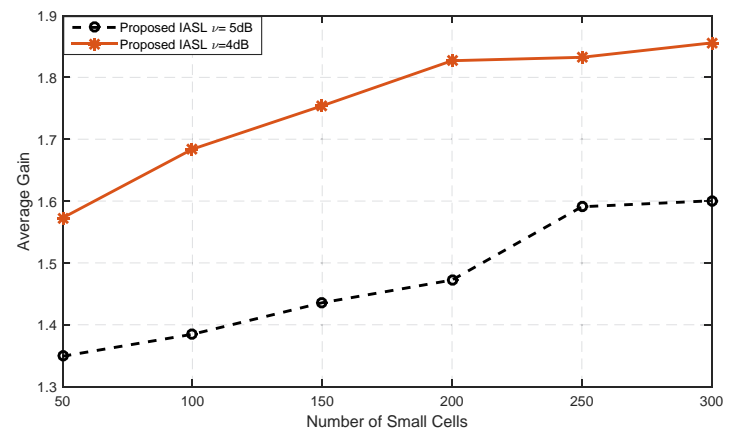

Fig. 2. Performance gain achieved by the proposed IASL scheme.

In Fig. 2, we describe the average performance gain with regard to the increasing number of small cells in the unit of node number $/ \mathrm{km}^{2}$. The average performance gain is defined as the ratio of the achieved performance before and after the implementations of the investigated schemes. The proposed IASL scheme can always obtain better performance compared to that of the non-cooperative scheme. However, the increasing rate of the gain decreases with the increasing number of small cells. Meanwhile, with the SINR threshold $v$ increasing, the average performance gain is more obvious.

\section{B. Traffic Offloading with Spectrum Leasing (TOSL)}

To mitigate the interference and balance the traffic, we provide another application of the formulated framework. The basic idea is small cells offloading parts of macrocell users, and in return, the macrocell should lease parts of its spectrum to the selected small cells in the partially shared channel deployment case. Therefore, we should solve the problems of how to formulate the coalition between macrocell and small cells, and how to implement the traffic offloading and spectrum leasing techniques in the formulated coalitions.

1) Details of Proposed TOSL Scheme: Different from the last application of the formulated coalition game-theoretic framework, and the coalition formation here is with respect to each channel. For each channel, we implement algorithm 2. Here, we also consider the downlink transmission of an OFDMA macrocell network, where multiple small cells are deployed. Initially, the initial coalition structure consists of the non-cooperative SeNBs and MeNB. All SeNBs partially share the orthogonal downlink channels of the MeNB. Meanwhile, SeNBs and the MeNB transmit the data via the maximum downlink power. Then, each MUE observes the interfering SeNBs that reuse the same subchannel and reports them to the MeNB. The MeNB constructs the interference list of the SeNBs in different subchannel. Based on the interference list, the MeNB negotiates with the SeNBs from the top of the list that whether to cooperate or not when compared to the revenue. The revenue is defined as $\pi=\frac{\sum R(\alpha, \beta, \psi)^{\delta}}{D^{(1-\delta)}}$, where the revenue is the net utility function between the achievable transmission rate and the average delay, where we still define the utility as the achievable transmission rate. $R$ is a function of achieved rate related to $\alpha, \beta$ and $\psi$, where the terms $\alpha$, and $\beta$ are the coefficient of spectrum leasing, the term $\psi$ is the coalition structure. $D$ and $\delta$ are the time delay and the tradeoff factor between the rate and the delay. If the revenue is increasing, the corresponding MUE is offloaded to the SeNB. Next, the SeNB will be leased to the time-slotted channel. Here, the whole time slot of a specific channel is assumed to be one unit "1". Then $1-\alpha$ is licensed to the MeNB, and $\alpha$ is partially shared between the MeNB and the SeNBs; this revenue function is also a convex function, that can be solved by the convex optimization. Finally, a stabilized coalition partition is reached.

2) Simulation and Numerical Results: Similar to the simulation setup of the proposed IASL algorithm, we verified the improved performance of the proposed TOSL scheme, where we used multiple small cells underlying the only macrocell as an example. We set the scenario as if there exist one small cell user associated with the small cell, which is used to simulate the traffic unbalance case and guarantees that all the femtocells can be the new associators selected by the specific macrocell users.

Fig. 3 illustrates the performance gain, where we use the non-cooperative one as the benchmark. Here, non-cooperative means that there is no coordination implementation between the macrocell and small cells. Meanwhile, we set two cases with different numbers of MUEs served by the macrocell when implementing the proposed TOSL scheme. 

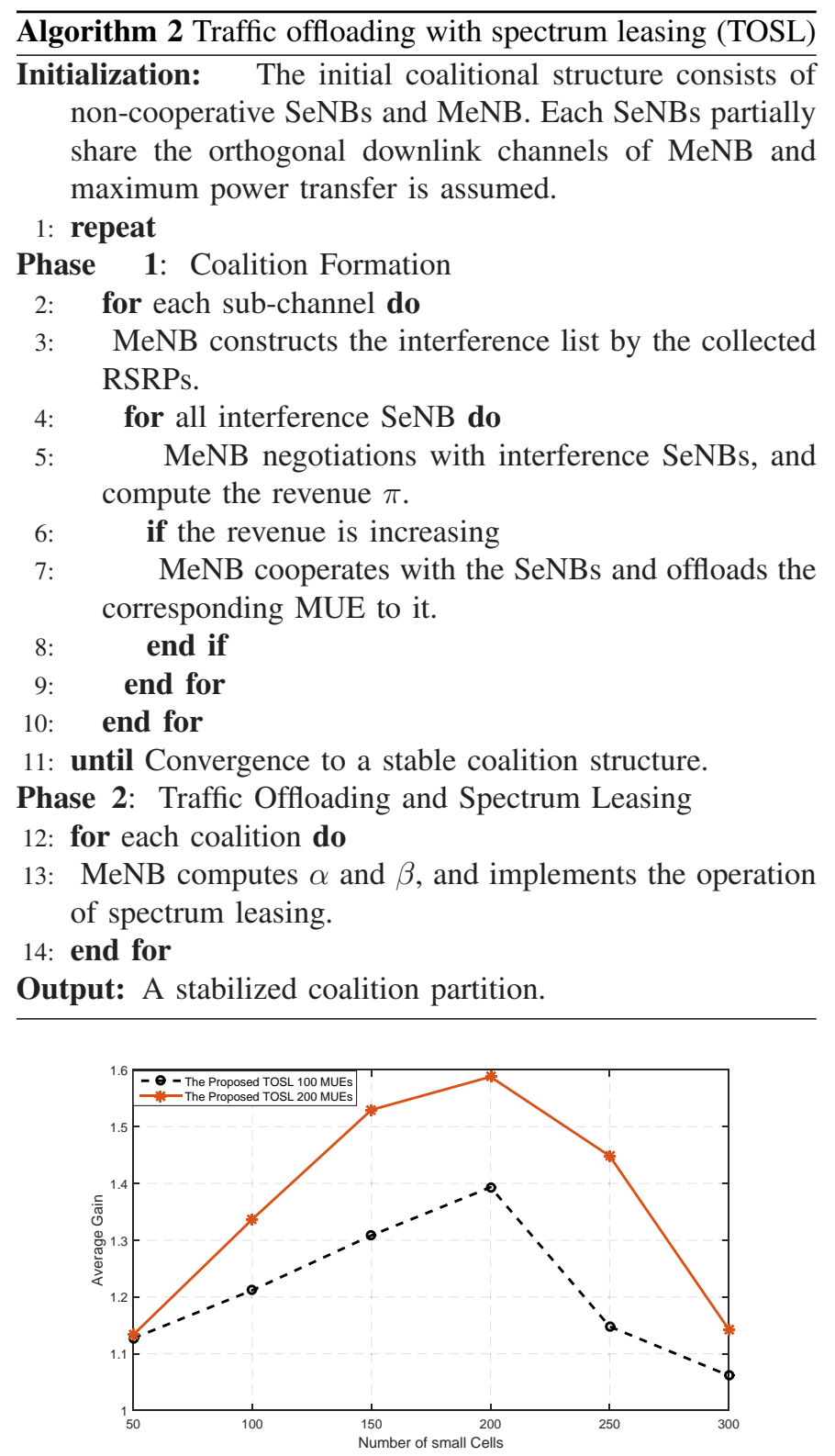

Fig. 3. Performance gain achieved by the proposed TOSL scheme.

We conclude from Fig. 3, that, the performance gain first increases and then decreases as the number of small cells increases, but TOSL can always achieve the performance improvement compared to the non-cooperative one. This is mainly due to the fact that there are limited available channels. Second, the performance gain is better when more MUEs are served by the macrocell. It is mainly because that more MUEs means more opportunities of traffic offloading to the femtocells. Meanwhile, we have assumed that each femtocell only serves one user, which means that all the offloaded MUEs can be re-associated with the femtocells.

\section{CONCLUSION}

To mitigate interference and balance traffic, we turned to cooperative games to explore the full cooperative gains among nodes. We presented the basics of coalition game and surveyed the latest applications. Moreover, we noted that cooperative incentive mechanism design was critical to form a suitable cooperative cluster, and thus we presented the cooperative framework with the spectrum leasing as the incentive mechanism. We also proposed schemes of IASL and TOSL under the presented framework. Simulation results verify the performance efficiency and show the improved performance of the proposed IASL and TOSL schemes. The presented framework can be extended to the non-orthogonal multiple access case and cognitive networks.

\section{REFERENCES}

[1] N. Panwar, S. Sharma, and A. K. Singh, "A survey on 5G: The next generation of mobile communication," Physical Communication, vol. 18, pp.64-84, Mar. 2016.

[2] M. Sheng, C. Yang, Y. Zhang, and J. Li, "Zone-based load balancing in LTE self-optimizing networks: a game-theoretic approach," IEEE Transactions on Vehicular Technology, vol. 63, no. 6, pp. 2916-2925, Jul. 2014.

[3] J. Wang, Z. Lv, Z. Ma, L. Sun, and Y. Sheng, "i-Net: new network architecture for 5G networks," IEEE Communications Magazine, vol. 53, no. 6, pp. 44-51, Jun. 2015.

[4] J. F. Monserrat, H. Droste, O. Bulakci, J. Eichinger, O. Queseth, M. Stamatelatos, H. Tullberg, V. Venkatkumar, G. Zimmermann, U. Dotsch, and A. Osseiran, "Rethinking the mobile and wireless network architecture: the METIS research into 5G," 2014 European Conference on Networks and Communications (EuCNC), Bologna, 2014, pp. 1-5.

[5] A. Al-Fuqaha, M. Guizani, M. Mohammadi, M. Aledhari, and M. Ayyash, "Internet of things: A survey on enabling technologies, protocols, and applications," IEEE Communications Surveys \& Tutorials, vol. 17, no. 4, pp. 2347-2376, Fourth-quarter. 2015.

[6] C. Yang, J. Li, Q. Ni, A. Anpalagan, and M. Guizani, "InterferenceAware Energy Efficiency Maximization in 5G Ultra-Dense Networks," IEEE Transactions on Communications, vol. 65, no. 2, pp. 728-739, Feb. 2017.

[7] F. Rebecchi, M. Dias de Amorim, V. Conan, A. Passarella, R. Bruno, and M. Conti, "Data Offloading Techniques in Cellular Networks: A Survey,", in IEEE Communications Surveys \& Tutorials, vol. 17, no. 2, pp. 580-603, Second-quarter. 2015.

[8] W. Saad, Z. Han, M. Debbah, A. Hjorungnes, and T. Basar, "Coalitional game theory for communication networks," IEEE Signal Processing Magazine, vol. 26, no. 5, pp. 77-97, Sep. 2009.

[9] F. Pantisano, M. Bennis, and W. Saad, "Interference alignment for cooperative femtocell networks: a game-theoretic approach," IEEE Transactions on Mobile Computing, vol. 12, no. 11, pp. 2233-2246, Nov. 2013.

[10] B. Guler and A. Yener, "Uplink interference management for coexisting MIMO femtocell and macrocell networks: an interference alignment approach," IEEE Transactions on Wireless Communications, vol. 13, no. 4, pp. 2246-2257, Mar. 2014.

[11] Y. Dong, M. Sheng, S. Zhang, and C. Yang. "Coalition based interference mitigation in femtocell networks with multi-resource allocation," 2014 IEEE International Conference on Communications (ICC), Sydney, 2014, pp. 2695-2700.

[12] C. Yang and J. Li, "Cooperative spectrum leasing to femtocells with interference compensation," 2014 IEEE 79th Vehicular Technology Conference (VTC Spring), Seoul, 2014, pp. 1-6.

[13] C. Yang, K. Guo, M. Sheng, J. Li, and J. Yue, "Energy-efficient capacity offload to smallcells with interference compensation," 2014 IEEE Wireless Communications and Networking Conference (WCNC), Turkey, 2014, pp. 1745-1749.

[14] H. Klessig, M. Gunzel, and G. Fettweis, "Increasing the capacity of large-scale HetNets through centralized dynamic data offloading," 2014 IEEE 80th Vehicular Technology Conference (VTC Fall), Vancouver, 2014 , pp. $1-7$

[15] 3GPP, "Evolved universal terrestrial radio access (E-UTRA); Further advancements for E-UTRA physical layer aspects," 3GPP Technical report (TR 36.814) v9.0.0, Mar. 2010. 


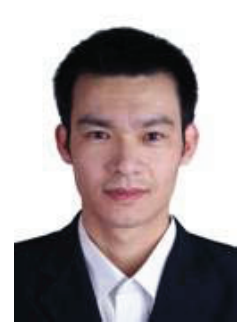

Chungang Yang (S'09, M'12) is currently an associate professor at Xidian University, where Dr. Yang leads the research team of "GUIDE, Game, Utility, Intelligent computing Design for Emerging communications". Dr. Yang received his Bachelor and Doctoral Degree at Xidian University, Xi'an, China, in 2006 and 2011, respectively. Between Sep. 2010 and March 2011, he held the visiting scholar position in the department of electrical and computer engineering at Michigan Technological University. Between March 2015 and March 2016, he held the visiting scholar position in the department of electrical and computer engineering at University of Houston. His research interests include resource and interference management, network optimization, and mechanism design for cognitive radio networks, heterogeneous cellular networks, and game theory for wireless communication and computing networks.

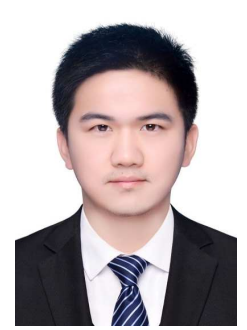

Jia Xiao received his Bachelor Degree degree in Communication Engineering at Xidian University in 2015. He is currently pursuing his Master Degree at Xidian University since 2015 . He works in the GUIDE research team, which is guided by Dr. Chungang Yang. His research interests include social-aware D2D communications, self-organizing ultra-dense small cell networks, and overlapping coalitional games.

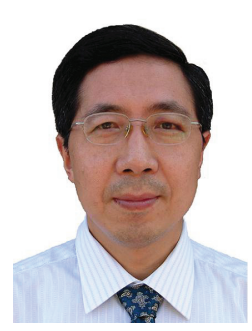

Jiandong Li (SM'05) was graduated from Xidian University with Bachelor Degree, Master Degree and $\mathrm{Ph}$. D in Communications and Electronic System respectively in 1982, 1985 and 1991 . He is with Xidian University from 1985, an associate professor from 1990 to 1994, professor from 1994, Ph.D student supervisor from 1995, and Dean of School of Telecommunication Engineering, Xidian University from 1997 respectively. Now, he also serves as Executive Vice Dean Graduate School, Xidian University.

Dr. $\mathrm{Li}$ is a senior member of IEEE and China Institute of Electronics (CIE) and the fellow of China Institute of Communication (CIC). He was the member of PCN specialist group for China 863 Communication high technology program between Jan. 1993 and Oct. 1994 and from 1999 to 2000. $\mathrm{He}$ is also the member of Communication specialist group for The Ministry of Information Industry. His current research interest and projects are funded by the 863 High Tech Project, NSFC, National Science Fund for Distinguished Young Scholars, TRAPOYT, MOE and MOI.

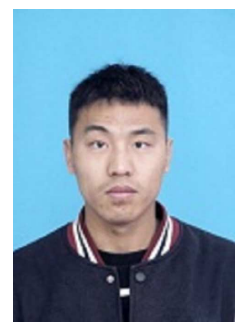

Xiaoqiang Shao received his Bachelor Degree degree in Communication Engineering at Xidian University in 2017 . He is currently pursuing his Master Degree at Xidian University since 2017. He works in the GUIDE research team, which is guided by Dr. Chungang Yang. His research interests include Nonorthogonal multiple access (NOMA) and cooperative game.

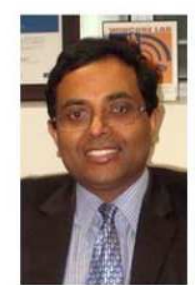

Alagan Anpalagan received the B.A.Sc. M.A.Sc. and $\mathrm{Ph} . \mathrm{D}$. degrees in Electrical Engineering from the University of Toronto, Canada. He joined the Department of Electrical and Computer Engineering at Ryerson University in 2001 and was promoted to Full Professor in 2010. He served the department as Graduate Program Director (2004-09) and the Interim Electrical Engineering Program Director (2009-10). During his sabbatical (2010-11), he was a Visiting Professor at Asian Institute of Technology and Visiting Researcher at Kyoto University. Dr. Anpalagan's industrial experience includes working at Bell Mobility, Nortel Networks and IBM Canada. Dr. Anpalagan directs a research group working on radio resource management (RRM) and radio access \& networking (RAN) areas within the WINCORE Lab. His current research interests include cognitive radio resource allocation and management, wireless cross layer design and optimization, cooperative communication, M2M communication, small cell networks, energy harvesting and green communications technologies. Dr. Anpalagan serves as Associate Editor for the IEEE Communications Surveys \& Tutorials (2012-) and Springer Wireless Personal Communications (2009-), and past Editor for IEEE Communications Letters (2010-13) and EURASIP Journal of Wireless Communications and Networking (2004-2009). He is a Senior Member of the IEEE.

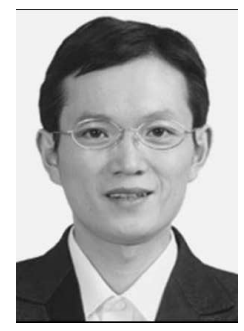

Qiang Ni (M'04-SM'08) received his Ph.D. degree in Engineering from Huazhong University of Science and Technology, Wuhan, in 1999. He is currently a Full Professor and the Head of Communication Systems Research Group at the School of Computing and Communications, Lancaster University, Lancaster, UK. His research interests include future generation communications and networking systems, energy and spectrum efficient green wireless communications, 5G, SDN, game theory, heterogeneous networks, cognitive radio network systems, ultradense networks, cloud networks, energy harvesting, IoTs, vehicular networks, big data analytics, etc. He had published more than 180 research papers in international journals and conferences. He was an IEEE 802.11 Wireless Standard Working Group Voting Member and a Contributor to the IEEE Wireless Standards.

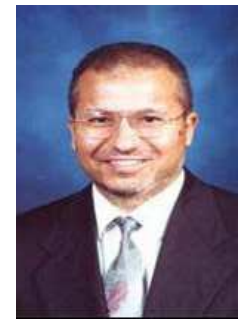

Mohsen Guizani (S'85-M'89-SM'99-F'09) received the B.S. (with distinction) and M.S. degrees in electrical engineering, the M.S. and Ph.D. degrees in computer engineering from Syracuse University, Syracuse, NY, USA, in 1984, 1986, 1987, and 1990, respectively. He is currently a Professor and the ECE Department Chair at the University of Idaho, USA. Previously, he served as the Associate Vice President of Graduate Studies, Qatar University, Chair of the Computer Science Department, Western Michigan University, and Chair of the Computer Science Department, University of West Florida. He also served in academic positions at the University of Missouri-Kansas City, University of Colorado-Boulder, Syracuse University, and Kuwait University. His research interests include wireless communications and mobile computing, computer networks, mobile cloud computing, security, and smart grid. He currently serves on the editorial boards of several international technical journals and the Founder and the Editor-in-Chief of Wireless Communications and Mobile Computing journal (Wiley). He is the author of nine books and more than 500 publications in refereed journals and conferences. He guest edited a number of special issues in IEEE journals and magazines. He also served as a member, Chair, and General Chair of a number of international conferences. He received the teaching award multiple times from different institutions as well as the best Research Award from three institutions. He received the Wireless Technical Committeeąŕs Recognition Award in 2017. He was the Chair of the IEEE Communications Society Wireless Technical Committee and the Chair of the TAOS Technical Committee. He served as the IEEE Computer Society Distinguished Speaker from 2003 to 2005. He is a Fellow of IEEE and a Senior Member of ACM. 